\title{
JourneyMap: Visualising the time-bound student Journey
}

\author{
Daniel Buzzo \\ University of the West of England \\ Coldharbour lane, Bristol, UK. \\ Daniel.buzzo@uwe.ac.uk
}

\author{
Philip Phelps \\ University of the West of England \\ Coldharbour lane, Bristol, UK. \\ Philip3.Phelps@uwe.ac.uk
}

\begin{abstract}
A student's view of higher education is often talked about as 'transformative' and as a 'journey' or 'pathway'. The student experience of university is time-bound, covering a clearly specified duration. From a learner's perspective the Higher Education journey begins at induction and ends at graduation. Through discrete and highly personal steps students move from one state, fresher, to another, graduate. This personal journey is explicitly linear, rather than the cyclic, annualised, time-perspective of the university.. The paper investigates how to visualise, in a temporal sense, this transformative journey. Incorporating necessary time bound events that appear in a students academic calendar and time table but in a student centric way that illustrates directly the journey the student takes and reinforces the time-bound, delineated nature of the student's HE experience. We document the results from development and present recommendations for future work
\end{abstract}

Time, temporality, interaction design, experience, research through design, visualisation

\section{INTRODUCTION}

From a temporally-orientated perspective a student's term-times, lectures, exams, assignments and other learning related temporally fixed events are way-points on a journey - each formative milestone passed will never be revisited. Along this journey reflective vistas and moments of selfevaluation frame the educational dialogue between student and institution. These moments can be vital in assisting a student in self-evaluation, identification of progress and generating meaningmaking and sense of achievement. All too often these important and instructional moments are lost to students. Particularly when mid-way through a learning journey they risk losing sight of the metaphorical hill they climb. Unable to see how far up they have come and that each step forward is one step closer to their goal.

The temporal representation seen by students of their academic life is of a calendar that repeats year after year, and is implicitly institutionally focused. It deals with the cyclic rhythms of the university as organisation. The temporal perspective of both institution and academic staff are at odds with the perspective of the student. The staff see repeating classes year after year with induction after induction to programmes. The organisation sees infrastructure and planning decades into the future with a past stretching back even further.
We proposed that the standard academic calendar/timetable is not conducive to reflective learning. The unique temporal perspective of the learner is transitional and transformative whereas the institution is grounded and cyclic. Our project worked with students to develop a range of alternative calendar/time event representations - to help learners think about the past and prepare for the future.

\section{TIME AS A LIVED EXPERIENCE}

The idea of time as a 'lived experience' has been discussed at length within mathematics, philosophy and physics and meta-physics for thousands of years. From the early thoughts of Parmenides (late sixth or early fifth century BC), though now surviving only in fragments of poems it appears that for him the phenomena of movement and change were simply appearances of a changeless, eternal reality. In the paradoxes of the Greek philosopher Zeno, (Ca 490-430 BC) we see this tension between our lived experience of the world and what logic may dictate of what underpins what we perceive. Nowhere is this tension more apparent than in the study of time, particularly in studying the lived experience of time and representations of time. More contemporary philosophers, in particular Husserl (1991), when he writes on the 'consciousness of internal time', and more recently Barad (2007) and the physicist Lee Smolin (2013) 
have argued about new interpretations of what time is and what it means to experience it. Le Poidevin (2009) when addressing the question 'how do we represent time' asks 'what is a representation and how do we perceive it'. Whilst an in-depth discussion of the complex and often competing theories of temporality, existence and perception are beyond the scope of this particular paper there are grounding arguments on visualisations of time, timelines and chronographs that have been of particular use. Notable as background to the design direction and theory of our project is the work of Aigner et al. $(2007,2011)$ Stephen Boyd Davis (2012, 2013) and in particular the large body of work from Edward Tufte (1983).

Rosenberg and Grafton (2010) allowed us insight into the history of temporal visualisation and of the changeable and often culturally specific nature of temporal representation. The work in the area of linguistics, temporality and spatiality (Fuhrman \& Boroditsky 2010, Boroditsky 2011) have been of particular use, supporting decisions on visualisation based on the western European student base we were designing with and for (Hendriks \& Boroditsky 2015). Whilst these references are not exhaustive by any measure as we have discussed in previous work in this area of temporal visualisation, and particularly calendar design projects (Buzzo \& Merendino 2015, Buzzo \& Jonas 2015, Buzzo $2014,2013)$ it must be remembered that the problem of time visualization is a 'wicked problem'. Originally identified by Rittel \& Webber (1973) the idea of a wicked problem that contains many factors, commonly human and cultural, that eludes a single solution, and that has many diverse possible resolutions has been documented extensively within the area of design. (Frankel \& Racine 2003, Buchanan 1992)

Several designers and researchers identify that the methodology of research through design is explicitly generative in nature and particularly suited to challenging, seemingly intractable problems. (Zimmerman, Forlizzi \& Evanson 2007, Gaver 2012) Taking this methodological approach of research through design we began with an exploratory investigation of how students visualise their individual journey through Higher Education. A guiding theory to our work was also provided by the ideas of technology as experience (Wright \& McCarthy 2004) and of situated action (Dourish 2004)

\subsection{Initial investigations: students}

The project began by conducting semi-structured interviews students at levels 1, 2 and 3 of an undergraduate Batchelor's degree in Science. The students were predominantly studying in the field of digital media and interaction design and so could be considered to have experience of the subject area. The initial interviews centered around the investigation of linguistic and spatial metaphors used by students. The Interviews prompted students to reflect on the time-limited period that they would spend within higher education and to investigate this as a progressive journey from one point to another rather than a cyclic activity. The reasoning behind this approach is based on the observation that the timescales used in discussion and representation of temporal activity from the university's perspective are often based upon permanent or long-term cyclic activities and repetition of events such as exams, evaluations, modules and courses. This is presented against the background of institutional timescales stretching back tens and in some instances hundreds of years. We contrasting these timescales and the temporal epistemology that underpins them with the lived student times-perspective and understanding. We felt this illustrated clearly how the idea of a time-limited student journey from induction to graduation may often be at odds with the language and representations they are presented with when institutional timescales and temporal-references are used.

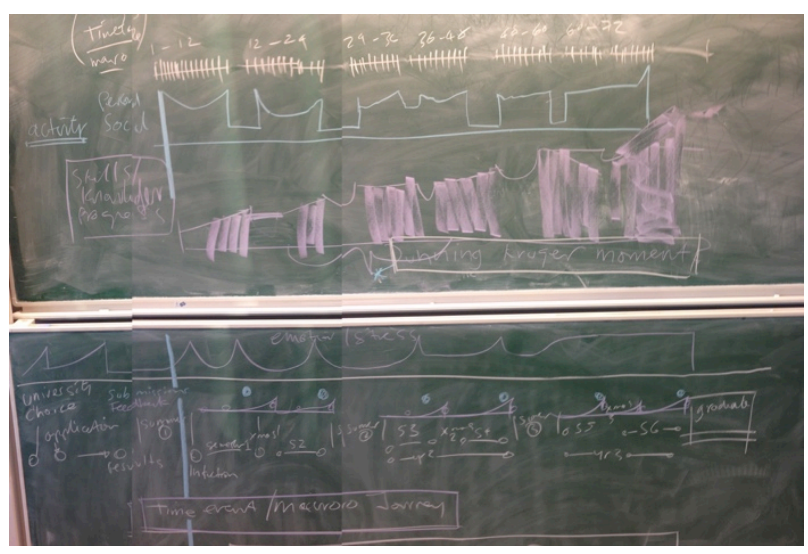

Figure 1: early collaborative work illustrating varieties of time events over the student HE journey, showing skill, emotion, chronology and events

The semi-structured interview questions also investigated students perceptions about educational transformation and improvement, particularly based upon ideas of progress. Language, metaphors and concepts that the students conveyed indicated that individually they understood the broader concept of higher education as a transformational journey of skills progression and personal development on a dayto-day scale. However it was apparent they found little to link their lived experience to temporal representations and activities with the broader idea of journey and educational progression.

When asked about repetition, activity, meaning and purpose within temporal activities, several students 
expressed surprise when confronted with the realisation that each workshop, seminar, session or lecture would only ever happen for them once in their educational journey. This realisation contrasted strongly with the language use where students expressed that much of what they did was repetitive, located in a cyclic educational process rather than a linear progressive one. When asked about calendar usage the answers from students were mixed, some regarding digital calendar usage as essential, helping them organise and plan their time around university, lectures and assignments. Other students appeared to do little in the way of formal temporal planning, occasionally referring to online timetables for information around scheduled activities such as room numbers for lectures. Some students appeared to reject, at an emotional level, structured temporal planning, seeing it as an authoritarian construct of the university and not something over which they had agency or input. Commonly students with less structured planning reported relying on their cohort for information about activities and also anecdotally for a sense of reflection or progress on their learning.

In more focused discussion on temporal planning students reflected that information about activities, events, and engagement was fragmented across many systems and presented in many different ways. Students also appeared to face challenges effectively reflecting on work they had done and learning goals they had achieved. This appeared reinforced by several student's apparent inability to visualise learning experiences such as seminars and lectures with subsequent coursework submission, assessment, and feedback as a connected series of events. This lack of connection with the elements in subject-centered learning appears to reinforce the disconnection between a subject of learning and the individual lectures, workshops, coursework, feedback and assessment related to the subject. This reinforced a feeling in some students of confusion and lack of purpose in their day-to-day and week to week activities. Some students appearing to struggle to see individual learning activities as leading towards any specific goal.

When considering the evaluation of educational learning objectives we used Bloom's Taxonomy (Bloom 1956) as a background to help investigate student achievement in terms of levels of complexity and mastery. Whilst Bloom's is not without it's critics it is widely regarded as a useful starting point for evaluation of learning. We were primarily interested in supporting Cognitive (knowledge based) and Affective (emotive) in selfevaluation and reflection through are also keen to investigate measures of Psychomotor (action based) in future work.
- Cognitive: mental skills (knowledge)

- Affective: growth in feelings or emotional areas (attitude or self)

- Psychomotor: manual or physical skills (skills)

\subsection{Student-centered, time-bound information}

When investigating the variety of data and information sources that carry time-bound or temporal information we discovered that almost every element the students come into contact with has macro or micro temporality. We discuss this distinction in greater depth in the section 'types of time events. When investigating the kinds of temporal data students deal with we were generally contrasting durations measured in hours at the micro scale and durations of months or tens of months at the macro scale. The primary temporal data we became interested in to visualise for Students included;

- Term and holiday dates

- Assignment dates

- Feedback on submissions, coursework and examinations

- Lectures, Workshops, and Seminars as part of programs of study (modules or courses)

- Special events such as guest lectures

In addition to this temporal data, we saw additional content that was explicitly tied to temporal events, e.g.

- Assignment coursework specifications

- Audio, video and textual feedback on submitted work

- Lecture slide sets, worksheets, example files, and video recordings

- Descriptions and additional material for special events (speaker descriptions, links etc)

\subsection{Design Exercises}

In our formal interview stage and investigation of some of the issues raised and discussed by students we researched the actuality of their temporal lived experience in their day-to-day and week to week study activities. We assembled a range of data types and associated content orientated information as core materials to be incorporated into possible prototypes. We engaged in a series of small, semi-structured participatory design exercises with small numbers of students. We presented students with a variety of temporal information we had identified alongside the temporally-linked content and asked them to go through a number of exercises generating spatialisations, hierarchies, affinities and 
associations between the elements. Of particular interest to us was how the students might structure the events and content in a linear or progression orientated fashion. Particularly if we felt these were specifically student-centered or perceptually orientated, reflecting the temporal lived-experience of students.

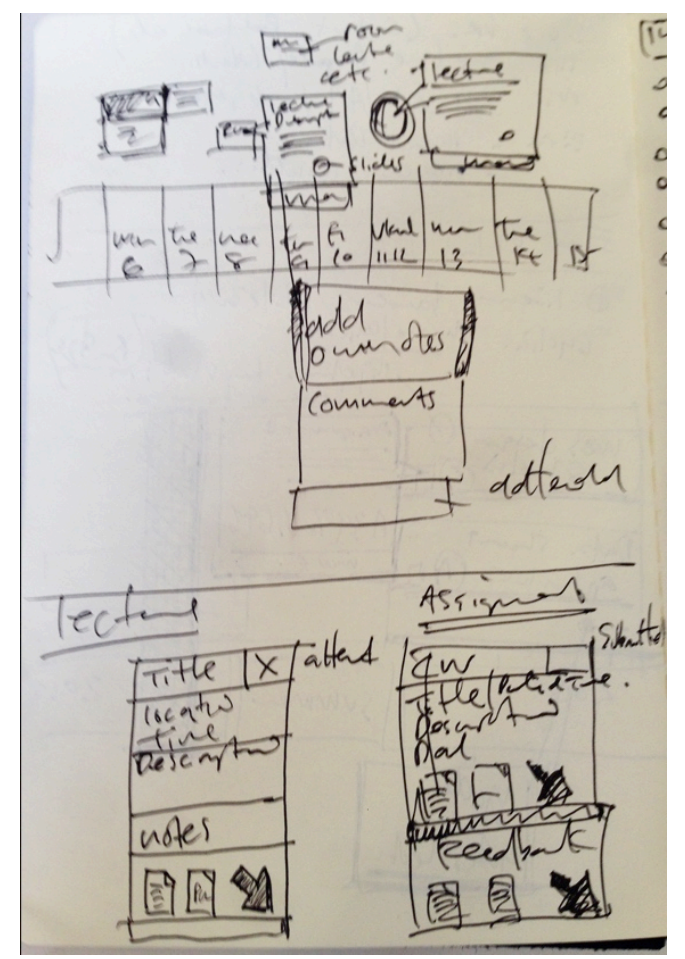

Figure 2: We generated a number of simple prototypes, commonly starting on paper. This illustrates ideas of how to visually associate 'micro scale' events and linked information and resources.

\subsubsection{Prototypes}

We generated a number of simple prototypes, commonly starting on paper and using iterative design processes and fast informal testing. This allowed us to develop ideas and analyse associations between durations, temporal events, reflection and linked informational content. During prototype generation we found that existing temporal representations were still extremely useful to students for orientation and sense making (perhaps due to their familiarity more than any other reason) and could potentially work effectively alongside other more adventurous or experimental illustrations of the student journey.

\subsubsection{Types of time events}

As we tested and evaluated prototypes we began to find greater clarity on the types of time events that students 'felt' in their lives at university. Time events ranged between what we term 'macro scale'. i.e. Events or situations such as each individual year of study, a run of a course or module in that year of study, longer term assignments or projects of coursework. These may cover eight weeks or more and include other long but time-bound events such as holidays. These contrasted with 'micro-scale' events.

The emphasis of macro time events was that they were seen by students more as loosely defined durations. The micro time-events that were considered included things that the students felt were explicitly pinned in time and that appeared to 'punctuate' macro events. Macro events were felt more as a duration than a specific point. With beginnings or ends that were described as being more 'gradual' or 'fuzzy'. Micro time-events were discussed by students using far more precise language and details. Example events listed by students included the beginning and ending of lectures, scheduled events on weekly timetables, meetings, and especially details of assignment submissions with hard deadlines.

\section{Macro time events \\ - year to year transition \\ - module runs \\ - assignments - preparation and execution \\ - holidays}

\section{Micro time events \\ - lectures, \\ - timetables \\ - meetings \\ - assignment submissions}

From our initial consultation, design work, testing, and evaluation of prototypes we found three objectives we hoped to reinforce within the student centered temporal visualisation.

\section{1) - Linking}

- Mentally and visually linking micro and macro time events

e.g to clearly relate an individual lecture to the wider goals of a course or programme of study, rather than it being a discreet 'disembodied' activity

\section{2) - Reflecting}

- Reflective progress through the journey of Higher Education -

- to clearly display how each day was a day further forward toward a goal.

- Reflection on improvement and transformation/personal growth and actualisation

- Allowing students to look back in time and see past achievements

\section{3) - Awareness of agency and control}


- Reflection on agency and control with student at the centre of process and situated action.

By putting the student at the centre of the temporal visualisation we sought to reinforce the perspective of the student being in control of their own learning and on their own educational journey.

- Reinforcing purpose and linking micro actions to macro results
To continually reinforce the idea of progress and change and to visually associate how daily decisions made by students affected larger scale goals and longer term activities.

\section{PROTOTYPE}

Having analysed data sources, content and also technical scope of delivery we developed an early operational prototype using live data sources. The prototype visualisation built upon efficacy and clarity we saw in the early stage design work using time-line orientated spatial structures. Working with predominantly European, English speaking subjects the design presented time running on a two-dimensional $x$-axis from left to right. (Rolke et al 2013, Fuhrman et al 2011) The user's perception of now being centered within the view with the past and performed events being to the left and future events goals and un-completed tasks to the right.

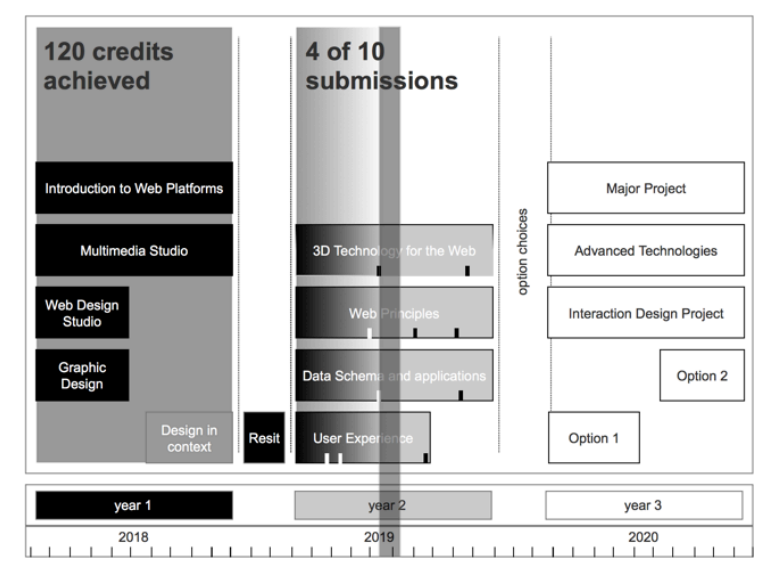

Figure 3: Early prototype using $x$ axis timeline showing wide (3 year) view of activities, illustrating the left/right future/past visualisation scenario.

Using time-line metaphors and map-style interactions allowed us to move away way from some of the restrictions of more traditional grid based, calendar style visualisation. The time-line and map metaphors allow background time-scales to be expanded and compressed to present greater or lesser granularity of durations and events being represented. This appeared to follow the language students had used to structure, navigate and describe the interrelation between macro and micro time events in their education experiences.

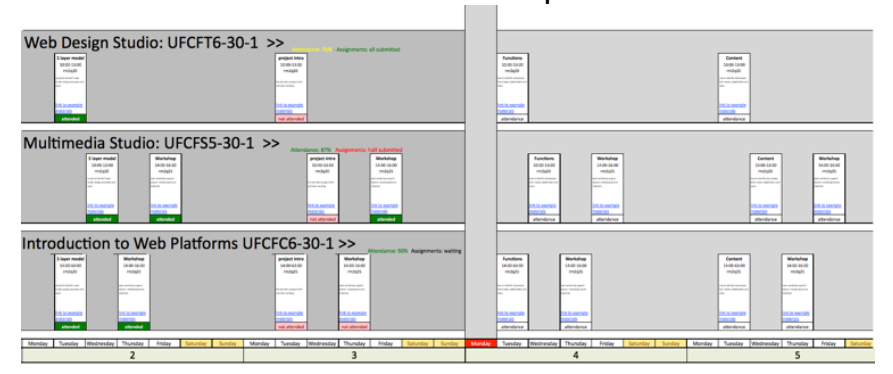

Figure 4: Medium resolution prototype timeline showing events grouped by course of study and indicating attendance: past events (red or green on dark grey background) on left and future/unresolved attendance (white on pale grey background) on right. 'Today' is indicated by grey bar with red highlight on day-name

\subsection{Using bounded time-lines}

Our approach to the design explicitly hinged on the students lived-experience of time in the context of a journey through higher education. To reinforce this the calendar was specifically time-bound to the length of study (usually three years) starting at the point of induction at level 1 , continuing to the day of graduation at level 3 and then stopping. The expectation is that should a students study be interrupted or extended the calendar would reflect this change in the length of the 'journey'. This linear, time-bound representation was seen to be crucial in communicating the base context of the design work, and of the subtle but important conceptual shift underpinning the project itself. One of shifting the centre of the representation to a student centered one rather than an institutionally focused one.

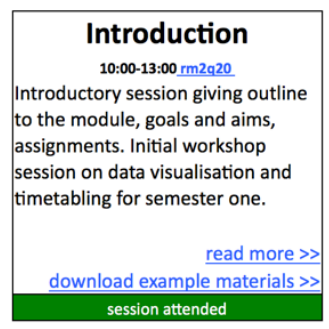

Figure 5: Example of individual event showing time, location, description, externally linked content and indication of attendance

The time-line data representation we have investigated seeks to work with current theory discussing the apparent contradictions in temporal representations. Specifically in the A series vs $B$ series time argument McTaggart (1908) proposes two views. One, of a tenseness universe (B series), one where all things are happening all the time and the idea of today, tomorrow and now is only relative and 'non-real' - as commonly represented by 
calendar view. This is juxtaposed with the lived experience of time, seen like a spotlight sweeping over events as they pass (A series). Robin Le Poidevin (2009) discusses these contrasting views in the context of asking what a perception or representation actually is. He contrasts the distinction between an egocentric and an objective representation and the problems when applying this view specifically to temporal perception. On the face of it our perception of time appears initillay egocentric, similar to spatial statements such as 'it is hot in here' or 'that mountain is further away than that tree'. Whilst the objective perception of space appears easy to do, 'the mountain is in this location, the tree is in this different location' he claims that the same cannot be said so easily of time, where ones own temporal perspective does not so easily appear to determine whether events are in the future or the past.

Traditional calendar representation appears to construct an objective representation of time. One where events written for a certain date do not change when the subjective or egocentric perception says that they have moved from the future to the past. The calendar may be more akin to a map in this sense. It's representation of space is appears an objective one within which we must clearly orientate ourselves, aware of our passage through the represented landscape. Tackling this simple but thorny philosophical and representational issue was a recurring theme in many of the early prototypes in our project.

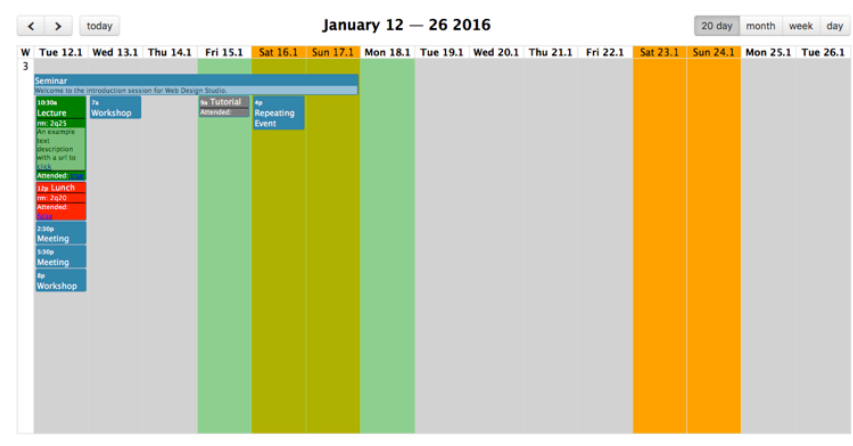

Figure 6: Early prototype built using HTML, CSS and JavaScript. Showing example data sources and active event attendance (shown as red and green events, events without recorded attendance showing in blue)

In our main development we addressed this issue in a simple fashion by changing the colour of all days and events that were objectively in the past. We indicated the viewer's location in a 'current' position in the temporal time-line and we also investigated the language used to describe future events. This attempt at strong-framing of the passage of time and progression of events through the bounded time-line was further reinforced by adding an extra data category to 'attendable' events - e.g. lectures seminars, workshops, and assignment submissions. Allowing us to record what the outcome was of events now in the past and leaving explicitly unresolved events in the future the outcome of which was yet to be resolved. This was an explicit attempt to address Objective 2) - that of encouraging reflection of progress though the educational journey and of Objective 3) linking micro-action to macro-purpose - linking attendance at individual events that were part of a longer process of small incremental stages rather than discrete events.

\subsection{Development}

The functional prototype student-journey time-line was built using client-side web technologies (HTML,CSS and JavaScript) to allow speed of development, standardisation and easy deployment to a variety of operating systems and platforms desktop, mobile etc. The fullcalendar.io JavaScript library was incorporated to provide simple calendaring operations and parsing of data in JSON (JavaScript Object Notation) retrieved from a remote Google Calendar. Fullcalendar.io is itself built on moment.js, an open source library designed to handle the complexities of low level date, event, timing and duration data. It also provides support for time-zones and a variety of events related to the iCalendar 'Internet Calendaring and Scheduling Core Object Specification' (RFC5545) for electronic calendaring data. Simple extensions within the ISO standard can accommodate event related information - e.g.; event type, event location, links and URLs of related information (slide-sets etc.). In addition to these properties we added a property to indicate whether the event had the capacity to be 'attended' and what the status of that was - attended, not attended (for events in the past), or yet to be determined. (for events in the future). These additional data elements were written into the existing non-standard property extension to the specification ' $x-$ '. In future development there appears to be the possibility to use the existing 'partstat-event' participant status with a new ' $x$-' status event of ' $x$-attended'. With 'null' indicating the event has not yet occurred and 'true' or 'false' indicating attendance status after the event has passed. It may additionally be possible to use the 'PERCENT-COMPLETE' properties of the 'partstattodo' property of calendar events, however the 'PERCENT-COMPLETE' property is explicitly associated with the 'TO-DO' event type and was originally specified to deal with tasks and activities rather than temporal/calendar events.

\subsubsection{Initial Testing}

Early evaluations of the functional prototype by students were conducted by short usage sessions and semi-structure reflective interviews. As of writing there have not yet been extend trials and 
results are based upon initial appraisals. The results have been used to form guidelines for future development.

\subsection{Future Development}

Future development includes options to switch to other, more traditional, calendar style or event-list displays. The may be a useful development for students, particularly in organising and visualising time-scales of days and weeks when integrating other tasks and activities such integrating study events with e.g. social engagements. This is currently available using modification to the code resources in the fullcalendar.io JavaScript library but will need some development work concerning which additional data and information sources will be displayed and how they may be visualised effectively in these more traditional formats.

An important part of the original motivation for the project was to encourage reflection and self awareness within students undertaking the higher education journey. From early design exercises it was proposed that students could add personal notes directly into calendar events, both for themselves, e.g. for making contemporaneous lecture notes and also for sharing such as asking questions or sharing additional resources that other attendees to the event would be able to see. Additional ability to make comments or notes in the form of diary style functionality was raised in early design sessions. Given the range of current practice and existing tool use it is suggested that linking to existing external sources be an easily investigated option - e.g. diary entries and note taking saved in Evernote and Google Docs software and social sharing via Facebook or twitter posts. These various 3rd party services all have web focused APls (Application Programming Interface) that can be used to write and recall information with relative ease. This step of continuing to integrate with external resources has a two-fold benefit. It integrates with user's existing systems and behaviour rather than attempting to introduce new work practices and it keeps the system being developed light and flexible.
Future technical development of the project should include closer integration with existing data sources. These include data from a number of disparate systems within the current university environment. Many systems exposed over the internal and external networks predominantly have some instance of a web service end point ( $A$ web service is a function that can be accessed by other programs over the web. A web service, as opposed to a web site is targeted at other programs rather than at humans) and given the correct configurations and, if necessary, security tokens the data can be integrated into the student JourneyMap project in a straightforward manner. The exposed web service endpoints serve a range of data formats and containers, usually XML (extensible markup language) or JSON (JavaScript Object Notation), that are easily accessible using standard web technologies. This will also help the project to be linked to university data sources such as the existing Virtual Learning Environment (VLE) from BlackBoard Inc., the internal timetabling and assignment system, and the submission and feedback systems. This would allow students to see, or link directly to, the bulk of data, content and information that has a temporal aspect or is related to temporal events. All of which is expressed in a time-bound, explicitly student entered visualisation that is designed to support the lived experience of the student journey.

\subsection{Conclusion}

Following these proposed developments we advocate a long-term adoptive study for more extensive testing of a robust stage 2 prototype. By combining a number of different measures, qualitative and quantitative we would hope to see where, why and how the temporal visualisation assists students in more clearly comprehending their time-bound journey through Higher Education. We would also hope to see clear areas for improvement and refinement, both in technical efficacy and functionality and in quality and effectiveness of the visual representation.

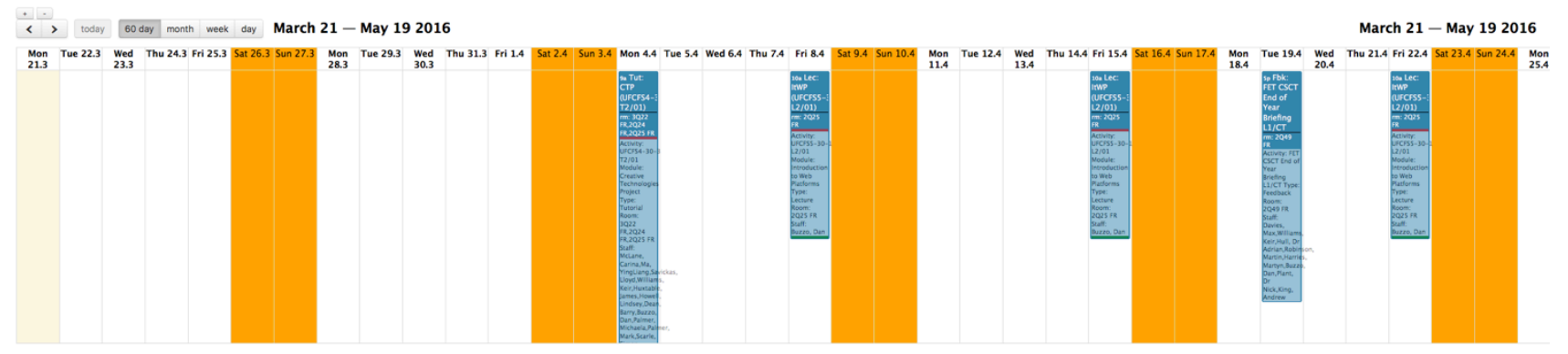

Figure 7: Iterated functional web based prototype. Showing live JSON data sources over 30 day range. 


\section{REFERENCES}

Aigner, W., Miksch, S., Müller, W., Schumann, H., \& Tominski, C. (2007). Visualizing time-oriented data-A systematic view. Computers \& Graphics, 31(3), 401-409. doi:10.1016/j.cag.2007.01.030

Aigner, W., Miksch, S., Schumann, H., \& Tominski, C. (2011). Visualization of Time-Oriented Data. London: Springer London. doi:10.1007/978-0-85729-079-3

Barad, Karen, (2007), Meeting the Universe Halfway: Quantum Physics and the Entanglement of Matter and Meaning, Durham and London: Duke University Press

Bloom, B. S. (1956). Taxonomy of Educational Objectives, Handbook I: The Cognitive Domain. New York: David McKay Co Inc.

Boroditsky, L. (2011). Chapter 20 - How Languages Construct Time. In S. Dehaene \& E. M. Brannon (Eds.), Space, Time and Number in the Brain (pp. 333-341). San Diego: Academic Press. Doi :http://dx.doi.org/10.1016/B978-0-12-385948-8.00020-7

Buchanan, R. (1992). Wicked Problems in Design Thinking. Design Issues, 8(2), 5-21. Retrieved from http://www.jstor.org/stable/1511637

Buzzo, D., \& Jonas, D. (2015). Designing for the impossible: Creating a mobile application to track time travel. In Electronic Visualisation and the Arts British Computer Society.

Buzzo, D., \& Merendino, N. (2015). Not all Days are Equal: Investigating the Meaning in the Digital Calendar. In Proceedings of the SIGCHI conference on Human factors in computing systems. Seoul, Korea: ACM Press.

Buzzo, D. (2013). The Shape of Time: Reconsidering time in the context of Pervasive Media. In Proceedings of the 1st Fascinate Conference Thoughtful Technology \& Beautiful Interfaces (pp. 1-3). Falmouth, UK.

Buzzo, D. (2013). Lost time never. In Proceedings of the 2013 Inputs-Outputs Conference: An Interdisciplinary Conference on Engagement in $\mathrm{HCl}$ and Performance. ACM - Association for Computing Machinery. Retrieved from http://www.inputs-outputs.org/

Buzzo, D. (2014). Time travel: Time dilation. In Electronic Visualisation and the Arts (pp. 170-176). British Computer Society.

Davis, S. B. (2012). History on the Line: Time as Dimension. Design Issues, 28(4), 4-17.

Davis, S. B. (2013). Time in Perspective: a visual approach to models of time. In $\mathrm{CHI}$ '13 CHI Conference on Human Factors in Computing Systems Paris, France - April 27 - May 02, 2013. MIT press.

Dourish, P. (2004). Where the Action Is: The Foundations of Embodied Interaction. MIT Press.

Frankel, L., \& Racine, M. (2003). The Complex Field of Research: for Design, through Design, and about Design.
Fuhrman, O., McCormick, K., Chen, E., Jiang, H., Shu, D., Mao, S., \& Boroditsky, L. (2011). How Linguistic and Cultural Forces Shape Conceptions of Time: English and Mandarin Time in 3D. Cognitive Science, 35(7). doi:10.1111/j.1551-6709.2011.01193.x

Hendricks, R. K., \& Boroditsky, L. (2015). New space time metaphors foster new mental representations of time Participants \& Analyses Experiment 1. Cognitive Science, 902-907.

Husserl, E., (1991). On the phenomenology of the consciousness of internal time (1893-1917),

Gaver, W. (2012). What Should We Expect From Research Through Design? Chi, 937-946. doi: $10.1145 / 2208516.2208538$

McCarthy, J., \& Wright, P. (2004). Technology as Experience. MIT Press. Retrieved from https://mitpress.mit.edu/index.php?q=books/technologyexperience

McTaggart, J. E. (1908). The Unreality of Time. Mind: $A$ Quarterly Review of Psychology and Philosophy, 17, 456-473. Retrieved from http://www.ditext.com/mctaggart/time.html

Poidevin, R. Le. (2009). The Images of Time: An Essay on Temporal Representation. Oxford University Press, USA.

Rittel, H. W. J., \& Webber, M. M. (1973). Dilemmas in a General Theory of Planning. Policy Sciences, (4), 155169. doi:doi:10.1007/bf01405730

Rolke, B., Fernández, S. R., Schmid, M., Walker, M., Lachmair, M., López, J. J. R., ... Vázquez, C. (2013). Priming the mental time-line: effects of modality and processing mode. Cognitive Processing, 14(3), 231-44. doi:10.1007/s10339-013-0537-5

Rosenberg, D. \& Grafton, A. (2010). Cartographies of Time: A History of the Timeline. Princeton Architectural Press. doi:10.5860/CHOICE.47-5799

Smolin, L. (2013). Time Reborn: From the crisis in physics to the future of the universe. London: Allen Lane. Fuhrman, O., \& Boroditsky, L. (2010). Cross-Cultural Differences in Mental Representations of Time: Evidence From an Implicit Nonlinguistic Task. Cognitive Science, 34(8), 1430-1451.

Tufte, E, R. (1983). The Visual Display of Quantitative Information (Cheshire, CT: Graphics Press, 1983), 10721.

Zimmerman, J., Forlizzi, J., and Evenson, S. (2007). Research through design as a method for interaction design research in $\mathrm{HCl}$, in: Proceedings of the SIGCHI conference on Human factors in computing systems, San Jose, California, p 493-502 